\title{
Functional approximation in Besov space using generalized Nörlund-Hausdorff product matrix
}

\author{
H.K. Nigam ${ }^{1 *}$ and Md. Hadish ${ }^{1}$
}

\section{"Correspondence:}

hknigam@cusb.ac.in

'Department of Mathematics, Central University of South Bihar, Gaya, India

\begin{abstract}
In the present work, a best approximation of a function $f$ in Besov space using the generalized Nörlund-Hausdorff $\left(N_{p q} \triangle_{H}\right)$ product means, for the two different cases $1<\sigma<\infty, \rho \geq 1,0 \leq \eta<v<2$ and $\sigma=\infty, 0 \leq \eta<v<2$, has been obtained. Our theorem generalizes the results of (Kyungpook Math. J. 50:545-556, 2010; Int. J. Appl. Math. 24(4):479-490, 2011; Nepal J. Sci. Technol. 14(2):117-122, 2013; Ultra Sci. Phys. Sci. 14(1): 53-58, 2002). Thus, the results of (Kyungpook Math. J. 50:545-556, 2010; Int. J. Appl. Math. 24(4):479-490, 2011; Nepal J. Sci. Technol. 14(2):117-122, 2013; Ultra Sci. Phys. Sci. 14(1): 53-58, 2002) become particular cases of our theorem. We also obtain some useful corollaries from our theorem.
\end{abstract}

MSC: 41A10; 41A25; 42B05; 42A50; 40G05; 40C05

Keywords: Approximation; Besov space; Fourier series; Functional; Generalized Nörlund means; Hausdorff means; Lipschitz space; Modulus of continuity; Modulus of smoothness

\section{Introduction}

Besov space serves to generalize more elementary functional spaces like Sobolov spaces, Lipschitz spaces, Hölder spaces, and generalized Hölder spaces. It is important to note that Besov space is effective at measuring regularity properties of functions.

Several researchers like those of [3,5-9] have obtained a degree of approximation of certain functions in different functional spaces such as Lipschitz space and Hölder spaces using single and product summability means. Therefore, in the present work, we obtain the degree of approximation of a function in a Besov space using generalized NörlundHausdorff $\left(N_{p q} \triangle_{H}\right)$ product means, which provide a more general estimate than those of [1-4].

\section{Preliminaries}

From [10] we define the following:

Let $C_{2 \pi}:=C[0,2 \pi]$ denote the Banach space of all $2 \pi$-periodic continuous function defined on $[0,2 \pi]$ under the supremum norm and

$$
L_{\rho}:=L^{\rho}[0,2 \pi]:=\left\{f:[0,2 \pi] \rightarrow \mathbb{R} ; \int_{0}^{2 \pi}|f(y)|^{\rho} d y<\infty, \rho \geq 1\right\}
$$


be the space of all $2 \pi$-periodic integrable functions.

The $L_{\rho}$-norm of a function $f$ is defined by

$$
\|f\|_{\rho}= \begin{cases}\left\{\frac{1}{2 \pi} \int_{0}^{2 \pi}|f(y)|^{\rho} d y\right\}^{\frac{1}{\rho}} & \text { for } 1 \leq \rho<\infty \\ {\operatorname{ess} \sup _{f \in(0,2 \pi)}|f(y)|} \text { for } \rho=\infty\end{cases}
$$

The modulus of continuity of a function $f$ in $L_{\rho}$ space is defined by

$$
w(f ; l)=\sup _{\substack{y, y+h \in[0,2 \pi] \\|h|<l}}|f(y+h)-f(y)| .
$$

The $k$ th order modulus of smoothness of a function $f$ in $L_{\rho}$ space is defined by

$$
\begin{aligned}
& w_{k}(f, l)_{\rho}=\sup _{0<h \leq l}\left\|\triangle_{h}^{k}(f, \cdot)\right\|_{\rho}, \quad l>0, \\
& \triangle_{h}^{k}(f, y)=\sum_{i=0}^{k}(-1)^{k-i}\left(\begin{array}{l}
k \\
i
\end{array}\right) f(y+i h), \quad k \in \mathbb{N} .
\end{aligned}
$$

\section{Remark 1}

(i) For $\rho=\infty, k=1$ and a continuous function $f$, the modulus of smoothness $w_{k}(f, l)_{\rho}$ reduces to the modulus of continuity $w(f, l)$.

(ii) For $0<\rho<\infty, k=1$ and a continuous function $f, w_{k}(f, l)_{\rho}$ becomes the integral modulus of continuity of first order $w(f, l)_{\rho}$.

Remark 2 If a function $f$ belongs to $C_{2 \pi}$ and $w(f, l)=O\left(l^{\nu}\right)$, for $0<v \leq 1$, then the function $f$ belongs to Lip $v$. If the function $f$ belongs to $L_{\rho}, 0<\rho<\infty$, and $w(f, l)_{\rho}=O\left(l^{\nu}\right), 0<v \leq 1$, then the function $f$ belongs to $\operatorname{Lip}(v, \rho)$.

If $\rho=\infty$ in class $\operatorname{Lip}(\nu, \rho)$ then $\operatorname{Lip}(v, \rho)$ class reduces to the class $\operatorname{Lip} v$. Thus,

$$
\operatorname{Lip} v \subseteq \operatorname{Lip}(v, \rho)
$$

Consider $v>0, k>v$ i.e., $k=[v]+1$, where $k$ is the smallest integer.

$$
\text { For } f \in L_{\rho} \text {, if }
$$

$$
w_{k}(f, l)_{\rho}=O\left(l^{\nu}\right), \quad l>0,
$$

then the function $f \in \operatorname{Lip}^{*}(\nu, \rho)$ (generalized Lipschitz class) and in this case the seminorm is given by

$$
|f|_{\text {Lip* }}=\sup _{l>0}\left(l^{-v} w_{k}(f, l)_{\rho}\right) .
$$

Thus,

$$
\operatorname{Lip}(v, \rho) \subseteq \operatorname{Lip}^{*}(v, \rho) .
$$


Remark 3 We are not representing here the definition of well-known Hölder spaces $H_{v}$ and $H_{v, \rho}$. The reader can consult [11] for detailed work on these spaces, It can be noted that $[11,12]$ :

(1) $H_{\nu} \subseteq H_{\eta} \subseteq C_{2 \pi}$ for $0<\eta \leq v \leq 1$ ( $H_{\nu}$ is a Banach space),

(2) $H_{v, \rho} \subseteq H_{\eta, \rho} \subseteq L_{\rho}$ for $0<\eta \leq v \leq 1, H_{v, \rho}$ is a Banach space for $\rho \geq 1$ and a complete $\rho$-normed space for $0<\rho<1$.

Let $v>0$ be given, and let $k=[v]+1$. For $0<\rho, \sigma \leq \infty$, the Besov space $B_{\sigma}^{v}\left(L_{\rho}\right)$ is a collection of all the functions ( $2 \pi$-periodic) $f \in L_{\rho}$ such that

$$
|f|_{B_{\sigma}^{v}\left(L_{\rho}\right)}:=\left\|w_{k}(f, \cdot)\right\|_{v, \sigma}= \begin{cases}\left(\int_{0}^{\pi}\left[l^{-v} w_{k}(f, l)_{\rho}\right]^{\sigma} \frac{d l}{l}\right)^{\frac{1}{\sigma}}, & 0<\sigma<\infty ; \\ \sup _{l>0}\left(l^{-v} w_{k}(f, l)_{\rho}\right), & \sigma=\infty,\end{cases}
$$

is finite [13].

Note 1 From (2) and (3) it is observed that, for $\sigma=\infty, B_{\infty}^{v}\left(L_{\rho}\right)=\operatorname{Lip}^{*}(v, \rho)$. Then the following cases are obtained:

(i) If we take $0<v \leq 1,0<\rho<\infty$, then $\operatorname{Lip}^{*}(v, \rho)$ reduces to the $\operatorname{Lip}(v, \rho)$ class.

(ii) If we take $\rho \rightarrow \infty$ then $\operatorname{Lip}(v, \rho)$ reduces to $\operatorname{Lip} v$ class.

It is observed that (4) is a seminorm if $1 \leq \rho, \sigma \leq \infty$ but a quasi-seminorm in other cases [10]. In this way, the quasi-norm for Besov space $B_{\sigma}^{v}\left(L_{\rho}\right)$ is given by

$$
\|f\|_{B_{\sigma}^{v}\left(L_{\rho}\right)}:=\|f\|_{\rho}+|f|_{B_{q_{1}}^{v}\left(L_{\rho}\right)}=\|f\|_{\rho}+\left\|w_{k}(f, \cdot)\right\|_{\nu, \sigma} .
$$

\section{Remark 4}

1. If $0<v<1$, the space $B_{\infty}^{v}\left(L_{\rho}\right)$ reduces to the space $H_{v, \rho}$ [14].

2. If $\rho=\infty=\sigma$ and $0<v<1$, the Besov space reduces to the space $H_{v}$ [15].

The $\delta$-order error of approximation of a function $f \in C_{2 \pi}$ is defined by

$$
E_{\delta}(f)=\inf _{t_{\delta}}\left\|f-t_{\delta}\right\|
$$

where $t_{\delta}$ is a trigonometric polynomial of degree $\delta$ [16].

If $E_{\delta}(f) \rightarrow 0$ as $\delta \rightarrow \infty$, the $E_{\delta}(f)$ is said to be the best approximation of $f$ [16].

Let $\sum_{\delta=0}^{\infty} u_{\delta}$ be an infinite series such that $s_{j}=\sum_{i=0}^{j} u_{i}$.

The $\delta$ th partial sum of the Fourier series (F. S.) is denoted by $s_{\delta}(f ; y)$ and is given by [16]

$$
s_{\delta}(f ; y)-f(y)=\frac{1}{2 \pi} \int_{0}^{\pi} \phi(y, l) \frac{\sin \left(\delta+\frac{1}{2}\right) l}{\sin \left(\frac{l}{2}\right)} d l .
$$

A Hausdorff matrix is a lower triangle matrix with entries

$$
h_{\delta, m}=\left(\begin{array}{c}
\delta \\
m
\end{array}\right) \triangle^{\delta-m} \mu_{m}
$$

where $\Delta \mu_{m}=\mu_{m}-\mu_{m+1}$ and $\Delta\left(\Delta^{\delta} \mu_{m}\right)=\Delta^{\delta+1} \mu_{m}$.

If $t_{\delta}^{\Delta_{H}}=\sum_{j=0}^{\delta} h_{\delta, m} s_{j}$ as $\delta \rightarrow \infty$, then the series $\sum_{\delta=0}^{\infty} u_{\delta}$ is said to be summable to the sum $s$ by the Hausdorff method ( $\triangle_{H}$ means). 
The Hausdorff matrix $H$ is regular, i.e., $H$ preserves the limit of each convergent sequence iff

$$
\int_{0}^{1}|d(v(z))|<\infty
$$

where the mass function $v \in B V[0,1], v(0+)=v(0)=0$, and $v(1)=1$. In this case, $\mu_{\delta}$ has the representation [17]

$$
\mu_{\delta}=\int_{0}^{1} z^{\delta} d v(z)
$$

Considering the two sequences $\left\{p_{\delta}\right\}$ and $\left\{q_{\delta}\right\}$, we write

$$
t_{\delta}^{N_{p q}}=\frac{1}{R_{\delta}} \sum_{k=0}^{\delta} p_{\delta-k} q_{k} s_{k} ; \quad R_{\delta}=\sum_{k=0}^{\delta} p_{k} q_{\delta-k} \neq 0 \quad \text { for all } \delta,
$$

then the generalized Nörlund means $\left(N_{p, q}\right)$ of the sequence $\left\{s_{\delta}\right\}$ is denoted by the sequence $t_{\delta}^{p q}$. If $t_{\delta}^{p q} \rightarrow s$, as $\delta \rightarrow \infty$ then the series $\sum_{\delta=0}^{\infty} u_{\delta}$ is said to be summable to $s$ by $N_{p, q}$ method and is denoted by $s_{\delta} \rightarrow s\left(N_{p, q}\right)[18]$.

The necessary and sufficient conditions for a $N_{p, q}$ method to be regular are

$$
\sum_{k=0}^{\delta}\left|p_{\delta-k} q_{k}\right|=O\left(\left|R_{\delta}\right|\right) \quad \text { and } \quad p_{\delta-k}=o\left(\left|R_{\delta}\right|\right) \quad \text { as } \delta \rightarrow \infty
$$

for every fixed $k \geq 0$ for which $q_{k} \neq 0$ [19].

The $N_{p, q}$ transform of the $t_{\delta}^{\Delta_{H}}$ transform defines the $N_{p q} \triangle_{H}$ product transform and its $\delta$ th partial sum is denoted by $t_{\delta}^{N_{p q} \Delta_{H}}$. Thus,

$$
\begin{aligned}
t_{\delta}^{N_{p q} \Delta_{H}} & =\frac{1}{R_{\delta}} \sum_{k=0}^{\delta} p_{\delta-k} q_{k} t_{k}^{\Delta_{H}} \\
& =\frac{1}{R_{\delta}} \sum_{k=0}^{\delta} p_{\delta-k} q_{k} \sum_{i=0}^{k} h_{k, i} s_{i} .
\end{aligned}
$$

If $t_{\delta}^{N_{p q} \Delta_{H}} \rightarrow s$ as $\delta \rightarrow \infty$, then $\sum_{\delta=0}^{\infty} u_{\delta}$ is summable by $N_{p q} \triangle_{H}$ product means to $s$. We have

$$
\begin{aligned}
s_{\delta} \rightarrow s & \Longrightarrow t_{\delta}^{\Delta_{H}} \rightarrow s \quad \text { as } \delta \rightarrow \infty, \triangle_{H} \text { method is obtained as regular } \\
& \Longrightarrow N_{p q}\left(t_{\delta}^{\triangle_{H}}\right)=t_{\delta}^{N_{p q} \Delta_{H}} \rightarrow s, \quad \text { as } \delta \rightarrow \infty, N_{p q} \text { means is obtained as regular } \\
& \Longrightarrow N_{p q} \triangle_{H} \quad \text { is obtained as regular. }
\end{aligned}
$$

\section{Note 2}

(i) $\triangle H$ means reduces to $C^{\alpha}$ means if $v(z)=\Pi_{k=1}^{\alpha} z^{k}, \alpha \geq 1$.

(ii) $\triangle H$ means reduces to $E^{q}$ if $h_{\delta, m}=\left(\begin{array}{c}\delta \\ m\end{array}\right) \frac{q^{\delta-m}}{(1+q)^{\delta}}, 0 \leq m \leq \delta$.

(iii) $N_{p, q}$ reduces to $N_{p}$ means if $q=1$. 
Remark 5 We define the following particular cases of the product means $N_{p q} \triangle_{H}$ :

(i) $N_{p, q} \triangle_{H}$ means reduces to $(N, p, q)(C, \alpha)$ or $N_{p q} C^{\alpha}$ means in view of Note 2(i).

(ii) $N_{p, q} \triangle_{H}$ means reduces to $(N, p, q)\left(E^{q}\right)$ or $N_{p q} E^{q}$ means in view of Note 2(ii).

(iii) $N_{p, q} \triangle_{H}$ means reduces to $N_{p} \triangle_{H}$ means in view of Note 2(iii).

\section{Note 3}

(i) Above particular case (i) in remark 5 is further reduced to $N_{p, q} C^{1}$ for $\alpha=1$.

(ii) Above particular case (ii) in remark 5 is further reduced to $N_{p, q} E^{1}$ for $q=1$.

(iii) Above particular case (iii) in remark 5 is further reduced to $N_{p} C^{\alpha}$ in view of Note 2(i) and then to $N_{p} C^{1}$ for $\alpha=1$.

(iv) Above particular case (iii) in remark 5 is further reduced to $N_{p} E^{q}$ in view of Note 2(ii) and then to $N_{p} E^{1}$ for $q=1$.

We write

$$
T_{\delta}(y)=t_{\delta}^{N_{p q} \Delta H}(y)-f(y)=\int_{0}^{\pi} \phi_{y}(u) M_{\delta}(u) d u,
$$

where

$$
\begin{aligned}
& M_{\delta}(u)=\frac{1}{2 \pi R_{\delta}} \sum_{k=0}^{\delta} p_{\delta-k} q_{k} \sum_{v=0}^{k} \int_{0}^{1}\left(\begin{array}{l}
k \\
v
\end{array}\right) z^{v}(1-z)^{k-v} d v(z) \frac{\sin \left(v+\frac{1}{2}\right) u}{\sin \left(\frac{u}{2}\right)} ; \\
& \phi_{y}(u)=f(y+u)+f(y-u)-2 f(y) ; \\
& \Phi(y, l, u)= \begin{cases}\phi_{y+l}(u)-\phi_{y}(u), & 0<v<1, \\
\phi_{y+l}(u)+\phi_{y-l}(u)-2 \phi_{y}(u), & 1 \leq v<2 ;\end{cases} \\
& \Upsilon_{\delta}(y, l)= \begin{cases}T_{\delta}(y+l)-T_{\delta}(y), & 0<v<1, \\
T_{\delta}(y+l)+T_{\delta}(y-l)-2 T_{\delta}(y), & 1 \leq v<2 .\end{cases}
\end{aligned}
$$

Remark 6 We prove the following additional results that will be used in the proof of our theorem.

(i) $\Upsilon_{\delta}(y, l)=\int_{0}^{\pi} M_{\delta}(u) \Phi(y, l, u) d u$,

(ii) $w_{k}\left(T_{\delta}, l\right)_{\rho}=\left\|\Upsilon_{\delta}(\cdot, l)\right\|_{\rho}$.

Proof (i) We have

$$
\begin{aligned}
\Upsilon_{\delta}(y, l) & = \begin{cases}T_{\delta}(y+l)-T_{\delta}(y), & 0<v<1, \\
T_{\delta}(y+l)+T_{\delta}(y-l)-2 T_{\delta}(y), & 1 \leq v<2,\end{cases} \\
& = \begin{cases}\int_{0}^{\pi}\left[\phi_{y+l}(u)-\phi_{y}(u)\right] M_{\delta}(u) d u, & 0<v<1, \\
\int_{0}^{\pi}\left[\phi_{y+l}(u)+\phi_{y-l}(u)-2 \phi_{y}(u)\right] M_{\delta}(u) d u, & 1 \leq v<2,\end{cases} \\
& =\int_{0}^{\pi} \Phi(y, l, u) M_{\delta}(u) d u .
\end{aligned}
$$


Proof (ii) By definition of $w_{k}(f, l)_{\rho}$, we have

$$
\begin{aligned}
w_{k}\left(T_{\delta}, l\right)_{\rho} & =\sup _{0<h \leq l}\left\|\triangle_{h}^{k}\left(T_{\delta}, \cdot\right)\right\|_{\rho} \\
& = \begin{cases}\sup _{0<h \leq l}\left\|T_{\delta}(\cdot+h)-T_{\delta}(\cdot)\right\|_{\rho}, & 0<v<1, \\
\sup _{0<h \leq l}\left\|T_{\delta}(\cdot+h)+T_{\delta}(\cdot-h)-2 T_{\delta}(\cdot)\right\|_{\rho}, & 1 \leq v<2,\end{cases} \\
& =\left\|\Upsilon_{\delta}(\cdot, l)\right\|_{\rho} .
\end{aligned}
$$

\section{Main theorem}

Theorem 3.1 For a function $f(2 \pi$-periodic and Lebesgue integrable) for $0 \leq \eta<v<2$, the best error approximation of $f$ in the Besov space $B_{\sigma}^{v}\left(L_{\rho}\right), \rho \geq 1,1<\sigma \leq \infty$ by $N_{p q} \triangle_{H}$ transform of its FS is given by

$$
\left\|T_{\delta}(\cdot)\right\|_{B_{\sigma}^{\eta}\left(L_{\rho}\right)}=O(1) \begin{cases}(\delta+1)^{-1}, & v-\eta-\sigma^{-1}>1, \\ (\delta+1)^{-v+\eta+\sigma^{-1}}, & v-\eta-\sigma^{-1}<1, \\ (\delta+1)^{-1}[\log (\delta+1) \pi]^{1-\sigma^{-1},}, & v-\eta-\frac{1}{\sigma}=1 .\end{cases}
$$

\section{Lemmas}

Lemma 4.1 If $\left\{p_{\delta}\right\}$ and $\left\{q_{\delta}\right\}$ are monotonic increasing and monotonic decreasing, respectively, then

$$
(\delta+1) p_{\delta} q_{0}=O\left(R_{\delta}\right)
$$

Proof

$$
\begin{aligned}
R_{\delta} & =\sum_{k=0}^{\delta} p_{\delta-k} q_{k}=p_{\delta} q_{0}+p_{\delta-1} q_{1}+\cdots+p_{0} q_{\delta} \\
& \geq p_{\delta} q_{0}+p_{\delta} q_{0}+\cdots+p_{\delta} q_{0} \\
& =(\delta+1) p_{\delta} q_{0} \\
(\delta & +1) p_{\delta} q_{0}=O\left(R_{\delta}\right) .
\end{aligned}
$$

Lemma 4.2 $M_{\delta}(u)=O(\delta+1)$ for $0<u \leq \frac{1}{\delta+1}$.

Proof For $0<u \leq \frac{1}{\delta+1}, \sin \left(\frac{u}{2}\right) \geq \frac{u}{\pi}, \sin \left(v+\frac{1}{2}\right) u \leq\left(v+\frac{1}{2}\right) u$ and $\sup _{0 \leq z \leq 1}\left|v^{\prime}(z)\right|=N$ we have

$$
\begin{aligned}
\left|M_{\delta}(u)\right| & =\left|\frac{1}{2 \pi R_{\delta}} \sum_{k=0}^{\delta} p_{\delta-k} q_{k} \sum_{v=0}^{k} \int_{0}^{1}\left(\begin{array}{l}
k \\
v
\end{array}\right) z^{v}(1-z)^{k-v} \frac{\sin \left(v+\frac{1}{2}\right) u}{\sin \left(\frac{u}{2}\right)} d v(z)\right| \\
& \leq \frac{1}{2 \pi R_{\delta}}\left|\sum_{k=0}^{\delta} p_{\delta-k} q_{k} \sum_{v=0}^{k} \int_{0}^{1}\left(\begin{array}{l}
k \\
v
\end{array}\right) z^{v}(1-z)^{k-v} \frac{\sin \left(v+\frac{1}{2}\right) u}{\frac{u}{\pi}} d v(z)\right| \\
& \leq \frac{1}{2 R_{\delta} u} \sum_{k=0}^{\delta} p_{\delta-k} q_{k} \sum_{v=0}^{k} \int_{0}^{1}\left(\begin{array}{l}
k \\
v
\end{array}\right) \frac{z^{v}}{(1-z)^{v}}(1-z)^{k} \sin \left(v+\frac{1}{2}\right) u|d v(z)|
\end{aligned}
$$




$$
\begin{aligned}
& \leq \frac{N}{4 R_{\delta} u} \sum_{k=0}^{\delta} p_{\delta-k} q_{k} \sum_{v=0}^{k} \int_{0}^{1}\left(\begin{array}{l}
k \\
v
\end{array}\right) A^{v}(2 v+1) \frac{u}{2}(1-z)^{k} d z, \quad \text { where } A=\frac{z^{v}}{(1-z)^{v}} \\
& =\frac{N}{4 \pi} \sum_{k=0}^{\delta} p_{\delta-k} q_{k} \int_{0}^{1}(1-z)^{k} d z \sum_{v=0}^{k}\left(\begin{array}{l}
k \\
v
\end{array}\right) A^{v}(2 v+1) \\
& =\frac{N}{4 \pi} \sum_{k=0}^{\delta} p_{\delta-k} q_{k} \int_{0}^{1}(1-z)^{k} d z\left\{2 \sum_{v=0}^{k} v\left(\begin{array}{l}
k \\
v
\end{array}\right) A^{v}+\sum_{v=0}^{k}\left(\begin{array}{l}
k \\
v
\end{array}\right) A^{v}\right\} \\
& =\frac{N}{4 \pi} \sum_{k=0}^{\delta} p_{\delta-k} q_{k} \int_{0}^{1}(1-z)^{k} d z\left\{2 \sum_{v=1}^{k} k\left(\begin{array}{l}
k-1 \\
v-1
\end{array}\right) A^{v}+(1+A)^{k}\right\} \\
& =\frac{N}{4 \pi} \sum_{k=0}^{\delta} p_{\delta-k} q_{k} \int_{0}^{1}(1-z)^{k} d z\left\{2 k(1+A)^{k-1}+(1+A)^{k}\right\} \\
& =\frac{N}{4 \pi} \sum_{k=0}^{\delta} p_{\delta-k} q_{k} \int_{0}^{1}[2 k(1-z)+1] d z \quad(\text { substituting the value of } A) \\
& =\frac{N}{4 \pi} \sum_{k=0}^{\delta} p_{\delta-k} q_{k}(k+1) \\
& \leq \frac{N(\delta+1)}{4 \pi} \sum_{k=0}^{\delta} p_{\delta-k} q_{k} \\
& =O(\delta+1) .
\end{aligned}
$$

Lemma 4.3 If $\left\{p_{\delta}\right\}$ and $\left\{q_{\delta}\right\}$ are monotonic increasing and monotonic decreasing sequences, respectively, then

$$
M_{\delta}(u)=O\left(\frac{1}{(\delta+1) u^{2}}\right) \quad \text { for } \frac{1}{\delta+1}<u \leq \pi .
$$

Proof For $\frac{1}{\delta+1}<u \leq \pi, \sin ^{2} \delta u \leq 1, \sin \left(\frac{u}{2}\right) \geq \frac{u}{\pi}$ and $\sup _{0 \leq z \leq 1}\left|v^{\prime}(z)\right|=N$

$$
\begin{aligned}
\left|M_{\delta}(u)\right| & =\left|\frac{1}{2 \pi R_{\delta}} \sum_{k=0}^{\delta} p_{\delta-k} q_{k} \sum_{v=0}^{k} \int_{0}^{1}\left(\begin{array}{l}
k \\
v
\end{array}\right) z^{v}(1-z)^{k-v} \frac{\sin \left(v+\frac{1}{2}\right) u}{\sin \left(\frac{u}{2}\right)} d \alpha(z)\right| \\
& \leq \frac{N}{2 R_{\delta} u}\left|\sum_{k=0}^{\delta} p_{\delta-k} q_{k} \operatorname{Im} \sum_{v=0}^{k} \int_{0}^{1}\left(\begin{array}{l}
k \\
v
\end{array}\right) z^{v}(1-z)^{k-v} e^{\ell\left(v+\frac{1}{2}\right) u} d z\right|
\end{aligned}
$$

First, we solve

$$
\begin{aligned}
& \operatorname{Im} \sum_{v=0}^{k} \int_{0}^{1}\left(\begin{array}{l}
k \\
v
\end{array}\right) z^{v}(1-z)^{k-v} e^{i\left(v+\frac{1}{2}\right) u} d z \\
& \leq \operatorname{Im} e^{i \frac{u}{2}} \int_{0}^{1} \sum_{v=0}^{k}\left(\begin{array}{l}
k \\
v
\end{array}\right) z^{v}(1-z)^{k-v} e^{i v u} d z \\
& =\operatorname{Im} e^{i \frac{u}{2}} \int_{0}^{1} \sum_{v=0}^{k}\left(\begin{array}{l}
k \\
v
\end{array}\right)(1-z)^{k-v}\left(z e^{i u}\right)^{v} d z
\end{aligned}
$$




$$
\begin{aligned}
& =\operatorname{Im} e^{i \frac{u}{2}} \int_{0}^{1}\left\{1+z\left(e^{i u}-1\right)\right\}^{k} d z \\
& =\operatorname{Im} \frac{e^{i(k+1) u}-1}{(k+1)\left(e^{i \frac{u}{2}}-e^{-i \frac{u}{2}}\right)} \\
& =\operatorname{Im} \frac{e^{i(k+1) u}-1}{(k+1) 2 i \sin \frac{u}{2}} \\
& =\operatorname{Im} \frac{\cos (k+1) u+i \sin (k+1) u-1}{2 i(k+1) \sin \frac{u}{2}} \\
& =\frac{\sin ^{2}(k+1) \frac{u}{2}}{(k+1) \sin \frac{u}{2}} .
\end{aligned}
$$

From (9) and (10), we have

$$
\begin{aligned}
\left|M_{\delta}(u)\right| & \leq \frac{N}{2 R_{\delta} u}\left|\sum_{k=0}^{\delta} p_{\delta-k} q_{k} \frac{\sin ^{2}(k+1) \frac{u}{2}}{(k+1) \sin \frac{u}{2}}\right| \\
& \leq \frac{N \pi}{2 R_{\delta} u^{2}}\left|\sum_{k=0}^{\delta} p_{\delta-k} q_{k} \frac{1}{k+1}\right| .
\end{aligned}
$$

Using Abel's lemma and Lemma 4.1, we have

$$
\begin{aligned}
\left|M_{\delta}(u)\right| & \leq \frac{N \pi}{2 R_{\delta} u^{2}}\left|\sum_{k=0}^{\delta-1}\left(p_{\delta-k} q_{k}-p_{\delta-k-1} q_{k+1}\right) \sum_{v=0}^{k} \frac{1}{v+1}+p_{0} q_{n} \sum_{k=0}^{\delta} \frac{1}{k+1}\right| \\
& \leq \frac{N \pi}{2 R_{\delta} u^{2}}\left[\sum_{k=0}^{v-1}\left|p_{\delta-k} q_{k}-p_{\delta-k-1} q_{k+1}\right|+p_{0} q_{\delta}\right] \max _{0 \leq k \leq m}\left|\sum_{k=0}^{m} \frac{1}{k+1}\right| \\
& \leq \frac{N \pi}{2 R_{\delta} u^{2}}\left[\left|p_{\delta} q_{0}-p_{0} q_{\delta}\right|+p_{0} q_{\delta}\right] \\
& \leq \frac{N \pi}{2 R_{\delta} u^{2}}\left(p_{\delta} q_{0}+2 p_{0} q_{\delta}\right) \\
& \leq \frac{3 p_{\delta} q_{o} N \pi}{2 R_{\delta} u^{2}} \\
M_{\delta}(u)= & O\left(\frac{1}{(\delta+1) u^{2}}\right) .
\end{aligned}
$$

Lemma 4.4 Let $1 \leq \rho \leq \infty$ and $0<v<2$. Iff $\in L_{\rho}$ then for $0<l, u \leq \pi$

(i) $\|\Phi(\cdot, l, u)\|_{\rho} \leq 4 w_{k}(f, l)_{\rho}$,

(ii) $\|\Phi(\cdot, l, u)\|_{\rho} \leq 4 w_{k}(f, u)_{\rho}$,

(iii) $\|\Phi .(u)\|_{\rho} \leq 2 w_{k}(f, u)_{\rho}$, where $k=[v]+1$.

Proof The proof of above lemma can be obtained along the same lines of the proofs of Lemma 2 in [20]. 
Lemma 4.5 Let $0 \leq \eta<v<2$. If $\in B_{\sigma}^{v}\left(L_{\rho}\right), \rho \geq 1,1<\sigma<\infty$, then

$$
\begin{gathered}
\text { (i) } \int_{0}^{\pi}\left|M_{\delta}(u)\right|\left(\int_{0}^{u} \frac{\|\Phi(\cdot, l, u)\|_{\rho}^{\sigma}}{l^{\eta \sigma}} \frac{d l}{l}\right)^{\frac{1}{\sigma}} d u=O(1)\left\{\int_{0}^{\pi}\left(u^{\nu-\eta} \mid\left(M_{\delta}(u)\right)^{\frac{\sigma}{\sigma-1}} d u\right\}^{1-\frac{1}{\sigma}},\right. \\
\text { (ii) } \int_{0}^{\pi}\left|M_{\delta}(u)\right|\left(\int_{u}^{\pi} \frac{\|\Phi(\cdot, l, u)\|_{\rho}^{\sigma}}{l^{\eta} \sigma} \frac{d l}{l}\right)^{\frac{1}{\sigma}} d u \\
=O(1)\left\{\int_{0}^{\pi}\left(u^{\nu-\eta+\frac{1}{\sigma}}\left|M_{\delta}(u)\right|\right)^{\frac{\sigma}{\sigma-1}} d u\right\}^{1-\frac{1}{\sigma}} .
\end{gathered}
$$

Proof The part of above lemma can be established along the same lines of the proofs of Lemma 2 in [20].

Lemma 4.6 ([20]) Let $0 \leq \eta<v<2$ and if $f \in B_{\sigma}^{v}\left(L_{\rho}\right), \rho \geq 1, \sigma=\infty$, then

$$
\sup _{0<l, u \leq \pi}\left(l^{-\eta}\|\Phi(\cdot, l, u)\|_{\rho}\right)=O\left(u^{\nu-\eta}\right) .
$$

\section{Proof of the main theorem}

\subsection{Case I: for $1<\sigma<\infty, \rho \geq 1,0 \leq \eta<v<2$}

Proof Following [16], we have

$$
s_{\delta}(f ; y)-f(y)=\frac{1}{2 \pi} \int_{0}^{\pi} \phi_{y}(l) \frac{\sin \left(\delta+\frac{1}{2}\right) l}{\sin \left(\frac{l}{2}\right)} d l .
$$

Denoting the Hausdorff matrix summability transform of $s_{\delta}(y)$ by $t_{\delta}^{\Delta_{H}}(y)$, we get

$$
\begin{aligned}
t_{\delta}^{\triangle_{H}}(y)-f(y) & =\sum_{m=0}^{\delta} h_{\delta, m}\left[s_{m}(y)-f(y)\right] \\
& =\sum_{m=0}^{\delta}\left(\begin{array}{c}
\delta \\
m
\end{array}\right) \Delta^{\delta-m} \mu_{m}\left\{\frac{1}{2 \pi} \int_{0}^{\pi} \phi_{y}(l) \frac{\sin \left(m+\frac{1}{2}\right) l}{\sin \left(\frac{l}{2}\right)} d l\right\} \\
& =\frac{1}{2 \pi} \int_{0}^{\pi} \phi_{y}(l) \sum_{m=0}^{\delta}\left(\begin{array}{c}
\delta \\
m
\end{array}\right) \Delta^{\delta-m}\left(\int_{0}^{1} z^{m} d \nu(z)\right) \frac{\sin \left(m+\frac{1}{2}\right) l}{\sin \left(\frac{l}{2}\right)} d l \\
& =\frac{1}{2 \pi} \int_{0}^{\pi} \phi_{y}(l) \sum_{m=0}^{\delta} \int_{0}^{1}\left(\begin{array}{c}
\delta \\
m
\end{array}\right) z^{m}(1-z)^{\delta-m} d v(z) \frac{\sin \left(m+\frac{1}{2}\right) l}{\sin \frac{l}{2}} d l .
\end{aligned}
$$

The $N_{p q}$ transform of $t_{\delta}^{\Delta_{H}}(y)$, denoted by $t_{\delta}^{N_{p q} \Delta_{H}}(y)$, is given by

$$
\begin{aligned}
& t_{\delta}^{N_{p q} \Delta_{H}}(y)-f(y) \\
& \quad=\frac{1}{R_{\delta}} \sum_{m=0}^{\delta} p_{\delta-k} q_{m}\left(\frac{1}{2 \pi} \int_{0}^{\pi} \phi_{y}(l) \sum_{v=0}^{m} \int_{0}^{1}\left(\begin{array}{c}
m \\
v
\end{array}\right) z^{v}(1-z)^{m-v} d v(z) \frac{\sin \left(v+\frac{1}{2}\right) l}{\sin \frac{l}{2}} d l\right) .
\end{aligned}
$$


Nigam and Hadish Journal of Inequalities and Applications

(2019) 2019:191

Page 10 of 17

Replacing $l$ by $u$

$$
\begin{aligned}
& =\int_{0}^{\pi} \phi_{y}(u) \frac{1}{2 \pi R_{\delta}} \sum_{m=0}^{\delta} p_{\delta-m} q_{m} \sum_{v=0}^{m} \int_{0}^{1}\left(\begin{array}{c}
m \\
v
\end{array}\right) z^{v}(1-z)^{m-v} d v(z) \frac{\sin \left(v+\frac{1}{2}\right) u}{\sin \left(\frac{u}{2}\right)} d u \\
& =\int_{0}^{\pi} \phi_{y}(u) M_{\delta}(u) d u .
\end{aligned}
$$

Let

$$
T_{\delta}(y)=t_{\delta}^{N_{p q} \Delta_{H}}(y)-f(y)=\int_{0}^{\pi} \phi_{y}(u) M_{\delta}(u) d u .
$$

Using the definition of the Besov norm given by (5), we have

$$
\left\|T_{\delta}(\cdot)\right\|_{B_{\sigma}^{\eta}\left(L_{\rho}\right)}=\left\|T_{\delta}(\cdot)\right\|_{\rho}+\left\|w_{k}\left(T_{\delta}, \cdot\right)\right\|_{\eta, \sigma} \cdot
$$

Now using (6) and Lemma 4.4(iii)

$$
\begin{aligned}
\left\|T_{\delta}(\cdot)\right\|_{\rho} & \leq \int_{0}^{\pi}\|\phi .(u)\|_{\rho}\left|M_{\delta}(u)\right| d u \\
& \leq \int_{0}^{\pi} 2 w_{k}(f, u)_{\rho}\left|M_{\delta}(u)\right| d u .
\end{aligned}
$$

Using Hölder's inequality and definition of Besov space given in (4), we get,

$$
\begin{aligned}
\left\|T_{\delta}(\cdot)\right\|_{\rho} \leq & 2\left\{\int_{0}^{\pi}\left(\left|M_{\delta}(u)\right| u^{\nu+\frac{1}{\sigma}}\right)^{\frac{\sigma}{\sigma-1}} d u\right\}^{1-\frac{1}{\sigma}}\left\{\int_{0}^{\pi}\left(\frac{w_{k}(f, u)_{\rho}}{u^{\nu+\frac{1}{\sigma}}}\right)^{\sigma} d u\right\}^{\frac{1}{\sigma}} \\
= & O(1)\left\{\int_{0}^{\pi}\left(\left|M_{\delta}(u)\right| u^{\nu+\frac{1}{\sigma}}\right)^{\frac{\sigma}{\sigma-1}} d u\right\}^{1-\frac{1}{\sigma}} \\
= & O\left[\left\{\int_{0}^{\frac{1}{\delta+1}}\left(\left|M_{\delta}(u)\right| u^{\nu+\frac{1}{\sigma}}\right)^{\frac{\sigma}{\sigma-1}} d u\right\}^{1-\frac{1}{\sigma}}\right. \\
& \left.+\left\{\int_{\frac{1}{\delta+1}}^{\pi}\left(\left|M_{\delta}(u)\right| u^{\nu+\frac{1}{\sigma}}\right)^{\frac{\sigma}{\sigma-1}} d u\right\}^{1-\frac{1}{\sigma}}\right] \\
= & R+S .
\end{aligned}
$$

Now using Lemma 4.2, we have

$$
\begin{aligned}
R & =O\left\{\int_{0}^{\frac{1}{\delta+1}}\left(\left|M_{\delta}(u)\right| u^{\nu+\frac{1}{\sigma}}\right)^{\frac{\sigma}{\sigma-1}} d u\right\}^{1-\frac{1}{\sigma}} \\
& =O\left[\int_{0}^{\frac{1}{\delta+1}}\left\{(\delta+1) u^{\nu+\frac{1}{\sigma}}\right\}^{\frac{\sigma}{\sigma-1}} d u\right]^{1-\frac{1}{\sigma}} \\
& =O\left\{(\delta+1)^{\frac{\sigma}{\sigma-1}} \int_{0}^{\frac{1}{\delta+1}} u^{\frac{\nu \sigma}{\sigma-1}+\frac{1}{\sigma-1}} d u\right\}^{1-\sigma^{-1}} \\
& =O\left\{\frac{1}{(\delta+1)^{v}}\right\} .
\end{aligned}
$$


Using Lemma 4.3, we have

$$
\begin{aligned}
S & =O\left\{\int_{\frac{1}{\delta+1}}^{\pi}\left(\left|M_{\delta}(u)\right| u^{v+\frac{1}{\sigma}}\right)^{\frac{\sigma}{\sigma-1}} d u\right\}^{1-\frac{1}{\sigma}} \\
& =O\left\{\int_{\frac{1}{\delta+1}}^{\pi}\left(\frac{1}{(\delta+1) u^{2}} u^{v+\frac{1}{\sigma}}\right)^{\frac{\sigma}{\sigma-1}} d u\right\}^{1-\frac{1}{\sigma}} \\
& =O\left\{\int_{\frac{1}{\delta+1}}^{\pi}\left(\frac{1}{\delta+1} \times u^{v+\frac{1}{\sigma}-2}\right)^{\frac{\sigma}{\sigma-1}} d u\right\}^{1-\frac{1}{\sigma}} \\
& =O(1) \begin{cases}(\delta+1)^{-1}, & v>1, \\
(\delta+1)^{-v}, & v<1, \\
(\delta+1)^{-1}[\log (\delta+1) \pi]^{1-\sigma^{-1}}, & v=1 .\end{cases}
\end{aligned}
$$

Combining (16)-(18), we have

$$
\left\|T_{\delta}(\cdot)\right\|_{\rho}=O(1) \begin{cases}(\delta+1)^{-1}, & v>1 \\ (\delta+1)^{-v}, & v<1 \\ (\delta+1)^{-1}[\log (\delta+1) \pi]^{1-\sigma^{-1}}, & v=1\end{cases}
$$

Using the generalized Minkowski inequality [21] repeatedly and Lemma 4.5, we get

$$
\begin{aligned}
\left\|w_{k}\left(T_{\delta}, \cdot\right)\right\|_{\eta, \sigma}= & {\left[\int_{0}^{\pi}\left(\frac{w_{k}\left(T_{\delta}, l\right)_{\rho}}{l^{\eta}}\right)^{\sigma} \frac{d l}{l}\right]^{\frac{1}{\sigma}} } \\
= & {\left[\int_{0}^{\pi}\left(\frac{\left\|\Upsilon_{\delta}(\cdot, l)\right\|_{\rho}}{l^{\eta}}\right)^{\sigma} \frac{d l}{l}\right]^{\frac{1}{\sigma}} } \\
\leq & \int_{0}^{\pi}\left|M_{\delta}(u)\right| d u\left(\int_{0}^{\pi} \frac{\|\Phi(\cdot, l, u)\|_{\rho}^{\sigma}}{l^{\eta \sigma}} \frac{d l}{l}\right)^{\sigma^{-1}} \\
\leq & {\left[\int_{0}^{\pi}\left|M_{\delta}(u)\right| d u\left\{\int_{0}^{u} \frac{\|\Phi(\cdot, l, u)\|_{\rho}^{\sigma}}{l \eta} \frac{d l}{l}\right\}^{\sigma^{-1}}\right] } \\
& +\left[\int_{0}^{\pi}\left|M_{\delta}(u)\right| d u\left\{\int_{u}^{\pi} \frac{\|\Phi(\cdot, l, u)\|_{\rho}^{\sigma}}{l^{\eta \sigma}} \frac{d l}{l}\right\}^{\sigma^{-1}}\right] \\
= & O(1)\left\{\int_{0}^{\pi}\left(u^{\nu-\eta}\left|M_{\delta}(u)\right|\right)^{\frac{\sigma}{\sigma-1}} d u\right\}^{1-\frac{1}{\sigma}} \\
& +O(1)\left\{\int_{0}^{\pi}\left(u^{\nu-\eta+\frac{1}{\sigma}}\left|M_{\delta}(u)\right|\right)^{\frac{\sigma}{\sigma-1}} d u\right\}^{1-\frac{1}{\sigma}} \\
= & O(1)\left(R_{1}+S_{1}\right) .
\end{aligned}
$$

Since $(a+b)^{\rho} \leq a^{\rho}+b^{\rho}$ for positive $a, b$ and $0<\rho \leq 1$ for $\rho=1-\frac{1}{\sigma}<1$, then

$$
\begin{aligned}
R_{1} & =\left\{\int_{0}^{\pi}\left(u^{\nu-\eta}\left|M_{\delta}(u)\right|\right)^{\frac{\sigma}{\sigma-1}} d u\right\}^{1-\sigma^{-1}} \\
& \leq\left\{\int_{0}^{\frac{1}{\delta+1}}\left(u^{\nu-\eta}\left|M_{\delta}(u)\right|\right)^{\frac{\sigma}{\sigma-1}} d u\right\}^{1-\sigma^{-1}}
\end{aligned}
$$




$$
\begin{aligned}
& +\left\{\int_{\frac{1}{\delta+1}}^{\pi}\left(u^{\nu-\eta}\left|M_{\delta}(u)\right|\right)^{\frac{\sigma}{\sigma-1}} d u\right\}^{1-\sigma^{-1}} \\
= & R_{11}+R_{12} .
\end{aligned}
$$

Using Lemma 4.2, we have

$$
\begin{aligned}
R_{11} & =O\left\{\int_{0}^{\frac{1}{\delta+1}}\left((\delta+1) u^{\nu-\eta}\right)^{\frac{\sigma}{\sigma-1}} d u\right\}^{1-\sigma^{-1}} \\
& =O\left\{(\delta+1)^{-\nu+\eta+\frac{1}{\sigma}}\right\} .
\end{aligned}
$$

Using Lemma 4.3, we have

$$
\begin{aligned}
R_{12} & =O\left\{\int_{\frac{1}{\delta+1}}^{\pi}\left(u^{\nu-\eta} \frac{1}{(\delta+1) u^{2}}\right)^{\frac{\sigma}{\sigma-1}} d u\right\}^{1-\sigma^{-1}} \\
& =O(1) \begin{cases}(\delta+1)^{-1}, & v-\eta-\sigma^{-1}>1, \\
(\delta+1)^{-\nu+\eta+\sigma^{-1}}, & v-\eta-\sigma^{-1}<1, \\
(\delta+1)^{-1}[\log (\delta+1) \pi]^{1-\sigma^{-1}}, & v-\eta-\frac{1}{\sigma}=1 .\end{cases}
\end{aligned}
$$

Now from (21) to (23), we get

$$
R_{1}=O(1) \begin{cases}(\delta+1)^{-1}, & v-\eta-\sigma^{-1}>1 \\ (\delta+1)^{-v+\eta+\sigma^{-1}}, & v-\eta-\sigma^{-1}<1 \\ (\delta+1)^{-1}[\log (\delta+1) \pi]^{1-\sigma^{-1}}, & v-\eta-\frac{1}{\sigma}=1\end{cases}
$$

Since $(a+b)^{\rho} \leq a^{\rho}+b^{\rho}$ for positive $a, b$ and $0<\rho \leq 1$ for $\rho=1-\frac{1}{\sigma}<1$, then

$$
\begin{aligned}
S_{1}= & \left\{\int_{0}^{\pi}\left(u^{\nu-\eta+\sigma^{-1}}\left|M_{\delta}(u)\right|\right)^{\frac{\sigma}{\sigma-1}} d u\right\}^{1-\sigma^{-1}} \\
\leq & \left\{\int_{0}^{\frac{1}{\delta+1}}\left(u^{\nu-\eta+\sigma^{-1}}\left|M_{\delta}(u)\right|\right)^{\frac{\sigma}{\sigma-1}} d u\right\}^{1-\sigma^{-1}} \\
& +\left\{\int_{\frac{1}{\delta+1}}^{\pi}\left(u^{\nu-\eta+\sigma^{-1}}\left|M_{\delta}(u)\right|\right)^{\frac{\sigma}{\sigma-1}} d u\right\}^{1-\sigma^{-1}} \\
= & S_{11}+S_{12} \text { say. }
\end{aligned}
$$

Using Lemma 4.2, we have

$$
\begin{aligned}
S_{11} & =O\left\{\int_{0}^{\frac{1}{\delta+1}}\left(u^{\nu-\eta+\sigma^{-1}}\left|M_{\delta}(u)\right|\right)^{\frac{\sigma}{\sigma-1}} d u\right\}^{1-\sigma^{-1}} \\
& =O\left\{\int_{0}^{\frac{1}{\delta+1}}\left(u^{\nu-\eta+\sigma^{-1}}(\delta+1)\right)^{\frac{\sigma}{\sigma-1}} d u\right\}^{1-\sigma^{-1}} \\
& =O\left\{(\delta+1)^{-\nu+\eta}\right\} .
\end{aligned}
$$


Using Lemma 4.3, we have

$$
\begin{aligned}
S_{12} & =O\left\{\int_{\frac{1}{\delta+1}}^{\pi}\left(u^{\nu-\eta+\frac{1}{\sigma}}\left|M_{\delta}(u)\right|\right)^{\frac{\sigma}{\sigma-1}} d u\right\}^{1-\sigma^{-1}} \\
& =O\left\{\int_{\frac{1}{\delta+1}}^{\pi}\left(u^{\nu-\eta+\frac{1}{\sigma}} \frac{1}{(\delta+1) u^{2}}\right)^{\frac{\sigma}{\sigma-1}} d u\right\}^{1-\sigma^{-1}} \\
& =O(1) \begin{cases}(\delta+1)^{-1}, & v-\eta>1, \\
(\delta+1)^{-v+\eta}, & v-\eta<1, \\
(\delta+1)^{-1}[\log (\delta+1) \pi]^{1-\sigma^{-1}}, & v-\eta=1 .\end{cases}
\end{aligned}
$$

Now, from (25)-(27), we get

$$
S_{1}=O(1) \begin{cases}(\delta+1)^{-1}, & v-\eta>1 \\ (\delta+1)^{-v+\eta}, & v-\eta<1 \\ (\delta+1)^{-1}[\log (\delta+1) \pi]^{1-\sigma^{-1}}, & v-\eta=1\end{cases}
$$

Combining (20), (24) and (28), we get

$$
w_{k}\left(T_{\delta}, \cdot\right) \|_{\eta, \sigma}=O(1) \begin{cases}(\delta+1)^{-1}, & v-\eta-\sigma^{-1}>1, \\ (\delta+1)^{-v+\eta+\sigma^{-1}}, & v-\eta-\sigma^{-1}<1, \\ (\delta+1)^{-1}[\log (\delta+1) \pi]^{1-\sigma^{-1}}, & \delta-\eta-\sigma^{-1}=1 .\end{cases}
$$

From (14), (19) and (29), we get

$$
\left\|T_{\delta}(\cdot)\right\|_{B_{\sigma}^{\eta}\left(L_{\rho}\right)}=O(1) \begin{cases}(\delta+1)^{-1}, & v-\eta-\sigma^{-1}>1, \\ (\delta+1)^{-v+\eta+\sigma^{-1}}, & v-\eta-\sigma^{-1}<1, \\ (\delta+1)^{-1}[\log (\delta+1) \pi]^{1-\sigma^{-1},}, & v-\eta-\frac{1}{\sigma}=1 .\end{cases}
$$

Case II: For $\sigma=\infty, 0 \leq \eta<v<2$.

$$
\left\|T_{\delta}(\cdot)\right\|_{B_{\infty}^{\eta}\left(L_{\rho}\right)}=\left\|T_{\delta}(\cdot)\right\|_{\rho}+\left\|w_{k}\left(T_{\delta}, \cdot\right)\right\|_{\eta, \infty} .
$$

Using (2) in (15),

$$
\begin{aligned}
\left\|T_{\delta}(\cdot)\right\|_{\rho} & \leq \int_{0}^{\pi} 2 w_{k}(f, u)_{\rho}\left|M_{\delta}(u)\right| d u \\
& =O(1)\left\{\int_{0}^{\frac{1}{\delta+1}}\left|M_{\delta}(u)\right| u^{\nu} d u+\int_{\frac{1}{\delta+1}}^{\pi}\left|M_{\delta}(u)\right| u^{\nu} d u\right\} \\
& =O(1)\left[R_{2}+S_{2}\right] .
\end{aligned}
$$


Using Lemma 4.2, we get

$$
\begin{aligned}
R_{2} & =\int_{0}^{\frac{1}{\delta+1}} u^{v}\left|M_{\delta}(u)\right| d u \\
& \leq \int_{0}^{\frac{1}{\delta+1}} u^{v}(\delta+1) d u=(\delta+1)^{-v} .
\end{aligned}
$$

Using Lemma 4.3, we get

$$
\begin{aligned}
S_{2} & =\int_{\frac{1}{\delta+1}}^{\pi} u^{v}\left|M_{\delta}(u)\right| d u \\
& \leq \frac{1}{\delta+1} \int_{\frac{1}{\delta+1}}^{\pi} u^{v-2} d u \\
& = \begin{cases}(\delta+1)^{-1}, & v>1, \\
(\delta+1)^{-v}, & v<1, \\
(\delta+1)^{-1} \log (\delta+1) \pi, & v=1 .\end{cases}
\end{aligned}
$$

From (32) and (34), we get

$$
\left\|T_{\delta}(\cdot)\right\|_{\rho}=O(1) \begin{cases}(\delta+1)^{-1}, & v>1 \\ (\delta+1)^{-v}, & v<1 \\ (\delta+1)^{-1} \log (\delta+1) \pi, & v=1 .\end{cases}
$$

Using the generalized Minkowski inequality [21] and Lemma 4.6, we get

$$
\begin{aligned}
\left\|w_{k}\left(T_{\delta}, \cdot\right)\right\|_{\eta, \infty} & =\sup _{l>0}\left(l^{-\eta} w_{k}\left(T_{\delta}, l\right)_{\rho}\right) \\
& =\sup _{l>0}\left(l^{-\eta}\left\|\Upsilon_{\delta}(\cdot, l)\right\|_{\rho}\right) \\
& =\sup _{l>0}\left[l^{-\eta}\left(\frac{1}{2 \pi} \int_{0}^{2 \pi}\left|\int_{0}^{\pi}\right| M_{\delta}(u)|\Phi(y, l, u) d u|^{\rho} d y\right)^{\frac{1}{\rho}}\right] \\
& \leq \sup _{l>0}\left[l^{-\eta}\left(\frac{1}{2 \pi}\right)^{\frac{1}{\rho}} \int_{0}^{\pi}\left\{\int_{0}^{2 \pi}\left|M_{\delta}(u)\right|^{\rho}|\Phi(y, l, u)|^{\rho} d y\right\}^{\frac{1}{\rho}} d u\right] \\
& =\sup _{l>0}\left[l^{-\eta} \int_{0}^{\pi}\|\Phi(\cdot, l, u)\|_{\rho}\left|M_{\delta}(u)\right| d u\right] \\
& =\int_{0}^{\pi}\left(\sup _{l>0} l^{-\eta}\|\Phi(\cdot, l, u)\|_{\rho}\right)\left|M_{\delta}(u)\right| d u \\
& =O(1) \int_{0}^{\pi} u^{\nu-\eta}\left|M_{\delta}(u)\right| d u \\
& =O(1)\left[\int_{0}^{\frac{1}{\delta+1}} u^{\nu-\eta}\left|M_{\delta}(u)\right| d u+\int_{\frac{1}{\delta+1}}^{\pi} u^{\nu-\eta}\left|M_{\delta}(u)\right| d u\right] \\
& =O(1)\left[R_{3}+S_{3}\right] .
\end{aligned}
$$


Using Lemma 4.2, we get

$$
\begin{aligned}
R_{3} & =\int_{0}^{\frac{1}{\delta+1}} u^{v-\eta}\left|M_{\delta}(u)\right| d u \\
& =O\left((\delta+1)^{\eta-v}\right) .
\end{aligned}
$$

Using Lemma 4.3, we get

$$
\begin{aligned}
S_{3} & =\int_{\frac{1}{\delta+1}}^{\pi} u^{v-\eta}\left|M_{\delta}(u)\right| d u \\
& =O(1) \frac{1}{\delta+1} \int_{\frac{1}{\delta+1}}^{\pi} u^{\nu-\eta-2} d u \\
& =O(1) \begin{cases}(\delta+1)^{-1}, & v-\eta>1, \\
(\delta+1)^{-v+\eta}, & v-\eta<1, \\
(\delta+1)^{-1} \log (\delta+1) \pi, & v-\eta=1 .\end{cases}
\end{aligned}
$$

From (36) to (38), we get

$$
\left\|w_{k}\left(T_{\delta}, \cdot\right)\right\|_{\eta, \infty}=O(1) \begin{cases}(\delta+1)^{-1}, & v-\eta>1 \\ (\delta+1)^{-v+\eta}, & v-\eta<1 \\ (\delta+1)^{-1} \log (\delta+1) \pi, & v-\eta=1\end{cases}
$$

Combining (31), (35) and (39) we obtain

$$
\left\|T_{\delta}(\cdot)\right\|_{B_{\sigma}^{\eta}\left(L_{\rho}\right)}=O(1) \begin{cases}(\delta+1)^{-1}, & v-\eta-\sigma^{-1}>1, \\ (\delta+1)^{-\nu+\eta+\sigma^{-1}}, & v-\eta-\sigma^{-1}<1, \\ (\delta+1)^{-1}[\log (\delta+1) \pi]^{1-\sigma^{-1}}, & v-\eta-\frac{1}{\sigma}=1 .\end{cases}
$$

\section{Corollaries}

Corollary 6.1 The error approximation of a function $f \in B_{\sigma}^{v}\left(L_{p}\right), \rho \geq 1,1<\sigma \leq \infty$ by $N_{p, q} C^{\alpha}$ means of its $F . S$ is given by

$$
\left\|T_{\delta}(\cdot)\right\|_{B_{\sigma}^{\eta}\left(L_{\rho}\right)}=O(1) \begin{cases}(\delta+1)^{-1}, & v-\eta-\sigma^{-1}>1 \\ (\delta+1)^{-v+\eta+\sigma^{-1}}, & v-\eta-\sigma^{-1}<1 \\ (\delta+1)^{-1}[\log (\delta+1) \pi]^{1-\sigma^{-1}}, & v-\eta-\frac{1}{\sigma}=1 .\end{cases}
$$

Corollary 6.2 The error approximation of a function $f \in B_{\sigma}^{v}\left(L_{\rho}\right), \rho \geq 1,1<\sigma \leq \infty$ by $N_{p q} E^{q}$ means of its FS is given by

$$
\left\|T_{\delta}(\cdot)\right\|_{B_{\sigma}^{\eta}\left(L_{\rho}\right)}=O(1) \begin{cases}(\delta+1)^{-1}, & v-\eta-\sigma^{-1}>1, \\ (\delta+1)^{-v+\eta+\sigma^{-1}}, & v-\eta-\sigma^{-1}<1, \\ (\delta+1)^{-1}[\log (\delta+1) \pi]^{1-\sigma^{-1},}, & v-\eta-\frac{1}{\sigma}=1 .\end{cases}
$$


Remark 7 Corollaries 6.1, 6.2 can be further reduced for $N_{p, q} C^{1}$ and $N_{p, q} E^{1}$ means, respectively in view of Note 3(i), (ii).

Corollary 6.3 The error approximation of $f \in B_{\sigma}^{v}\left(L_{\rho}\right), \rho \geq 1,1<\sigma \leq \infty$ by $N_{p} \triangle_{H}$ means of its F. S is given by

$$
\left\|T_{\delta}(\cdot)\right\|_{B_{\sigma}^{\eta}\left(L_{\rho}\right)}=O(1) \begin{cases}(\delta+1)^{-1}, & v-\eta-\sigma^{-1}>1, \\ (\delta+1)^{-v+\eta+\sigma^{-1}}, & v-\eta-\sigma^{-1}<1, \\ (\delta+1)^{-1}[\log (\delta+1) \pi]^{1-\sigma^{-1},}, & v-\eta-\frac{1}{\sigma}=1 .\end{cases}
$$

Remark 8 Corollary 6.3 can be further reduced for $N_{p} C^{\alpha}, N_{p} C^{1}, N_{p} E^{q}, N_{p} E^{1}$ in view of Note 3(iii), (iv).

\section{Particular cases}

7.1. Using Note 1 (ii) and Note 3(iv) and by putting $\eta=0$ in our result, our theorem becomes a particular case of main theorem of [4].

7.2. Using Note 1(i) and Note 3(iii) and by putting $\eta=0$ in our result, our main theorem becomes a particular case of main theorem of [1].

7.3. Using Note 1 (i) and Note 3(i) by putting $\eta=0$ in our result, our main theorem becomes a particular case of main theorem of [3].

7.4. If $\xi(t)=t^{\alpha}$ then $\operatorname{Lip}((\xi(t), r)$ class reduces to $\operatorname{Lip}(\alpha, r)$ class, where $\xi(t)$ is a positive increasing function and $r \geq 1$. Further as $r \rightarrow \infty$ in $\operatorname{Lip}(\alpha, r)$ class reduces to $\operatorname{Lip} \alpha$ class. Thus, using this argument in [2] and putting $\eta=0$ in our result, our main theorem becomes a particular case of [2].

\section{Conclusion}

In the review literature, it has been observed that many results have been obtained by the researchers on the degree of approximation of certain functions in different functional spaces like Lipschitz space, Hölder spaces etc. using the trigonometric Fourier approximation method. Since the Besov space generalizes more elementary functions as mentioned above and this space is very effective in measuring regularity properties of the function, this space has a wide range of applications in different areas of engineering and in mathematics in general and in analysis in particular.

Motivated by the usefulness of the Besov space in approximating the error of a certain function, in the present work we estimate the error of a function $f$ in Besov space using a generalized Nörlund-Hausdorff $\left(N_{p q} \triangle_{H}\right)$ product matrix, our result generalizes several previously known results obtained by using a Lipschitz space. Thus, the results of [1-4] become particular cases of our theorem. Some useful results are also deduced in the form of corollaries from our theorem.

Some other studies regarding the modulus of the smoothness of functions using different function spaces may be performed in future work.

\section{Acknowledgements}

The first author expresses his gratitude towards his mother for her blessings. The first author also expresses his gratitude towards his father in heaven, whose soul is always guiding and encouraging him. The second author is thankful to the University Grants Commission, India, for providing a Junior Research fellowship (JRF) to carry out the present work as a part of Ph.D, degree. The second author also expresses his gratitude towards his parents for blessings. Both the authors are also grateful to the Hon'ble vice-chancellor, Central University of South Bihar, for motivation to this work. 
Funding

Not applicable.

\section{Availability of data and materials}

Not applicable.

\section{Competing interests}

The authors declare that they have no competing interests.

\section{Authors' contributions}

All authors contributed equally to the writing of this paper. HKN framed the problems. HKN and MH carried out the results and wrote the manuscripts. All the authors read and approved the final manuscripts.

\section{Publisher's Note}

Springer Nature remains neutral with regard to jurisdictional claims in published maps and institutional affiliations.

Received: 6 April 2019 Accepted: 5 June 2019 Published online: 11 July 2019

\section{References}

1. Nigam, H.K., Sharma, A.: On approximation of functions belonging to $\operatorname{Lip}(\alpha, r)$ and to weighted $w\left(L_{r}, \xi(t)\right)$ class by product means. Kyungpook Math. J. 50, 545-556 (2010)

2. Nigam, H.K., Sharma, K.: Degree of approximation of a function belonging to $\operatorname{Lip}(\xi(t), r)$ class by $(N, p, q)(E, 1)$ product means of Fourier series. Int. J. Appl. Math. 24(4), 479-490 (2011)

3. Kushwaha, J.K., Dhakal, B.P.: Approximation of a function belonging to $\operatorname{Lip}(\alpha, r)$ class by $N_{p, q}$. $C_{1}$ summability method of its Fourier series. Nepal J. Sci. Technol. 14(2), 117-122 (2013)

4. Lal, S., Nigam, H.K.: On degree of approximation of a Lipschitz function by $\left(N, p_{n}\right)(E, 1)$ means of its Fourier series. Ultra Sci. Phys. Sci. 14(1), 53-58 (2002)

5. Rhaodes, B. E: On the degree of approximation of functions belonging to Lipschitz class by Hausdorff means of its Fourier series. Tamkang Journal of Mathematics 34(2), 245-247 (2003)

6. Dhakal, B.P.: Approximation of functions belonging to Lip $\alpha$ class by matrix-Cesàro summability method. Int. Math. Forum 5(35), 1729-1735 (2010)

7. Nigam, H.K., Hadish, M.: Best approximation of functions in generalized Hölder class. J. Inequal. Appl. 2018,276 (2018)

8. Lal, S., Mishra, A.: The method of summation $(E, 1)\left(N, p_{n}\right)$ and trigonometric approximation of function in generalized Holder metric. J. Indian Math. Soc. 80(1-2), 87-98 (2013)

9. Singh, U., Kumar Srivastava, S.: Degree of approximation of functions belonging to certain Lipschits class by $C^{1} . T$ operator. Asian-Eur. J. Math. 07, 1450064 (2014)

10. DeVore, R.A., Lorentz, G.G.: Constructive Approximation. Springer, New York (1993)

11. Nayak, L., Das, G., Ray, B.K.: An estimate of the rate of convergence of Fourier series in generalized Hölder metric by deffered Cesàro mean. J. Math. Anal. Appl. 420, 563-575 (2014)

12. Maddox, I.J.: Elements of Functional Analysis. Cambridge University Press, Cambridge (1971)

13. Wojtaszczyk, P.: A Mathematical Introduction to Wavelets. London Mathematical Society Student Texts, vol. 37. Cambridge University Press, New York (1997)

14. Das, G., Ghosh, T., Ray, B.K.: Degree of approximation by their Fourier series in generalized Hölder metric. Proc. Indian Acad. Sci. Math. Sci. 106, 139-153 (1996)

15. Prössdorf, S: Zur Konvergenz der Fourierreihen Hölder stetiger Funktionen. Math Nachr. 69 , 7-14 (1975)

16. Zygmund, A.: Trigonometric Series, 3rd rev. edn. Cambridge Univ. Press, Cambridge (2002)

17. Boos, J., Cass, P.: Classical and Modern Methods in Summability. Oxford University Press, New York (2000)

18. Borwein, D.: On products of sequences. J. Lond. Math. Soc. 33, 352-357 (1958)

19. Hardy, G.H.: Divergent Series. Oxford University Press, London (1949)

20. Mohanty, H., Das, G., Ray, B.K.: Degree of approximation of Fourier series of functions in Besov space by $\left(N, p_{n}\right)$ mean. J. Orissa Math. Soc. 30(2), 13-34 (2011)

21. Chui, C.K.: An Introduction to Wavelets. Wavelet Analysis and Its Applications, vol. 1. Academic Press, San Diego (1992)

\section{Submit your manuscript to a SpringerOpen ${ }^{0}$ journal and benefit from:}

- Convenient online submission

- Rigorous peer review

- Open access: articles freely available online

- High visibility within the field

Retaining the copyright to your article

Submit your next manuscript at $>$ springeropen.com 